\title{
On the Space-Time Description of Interference Phenomena in Nuclear Reactions with Three Particles in the Final Channel
}

\author{
V.S. Olkhovsky* and S.A. Omelchenko
}

Institute for Nuclear Research of NASU, Kiev-0650, Ukraine

\begin{abstract}
The aim of the present work is to show, on a correct self-consistent base of the space-time description of the nuclear processes with three particles in the final channel, the validity of the former approach, generalizing the results, obtained for the space-time description of the nuclear processes with two-particle channels earlier in some of our recent papers.

Pacs Numbers: 24.50.+g; 25.40.Ny; 25.70.-Pq.
\end{abstract}

Keywords: Interference phenomena, recoil system, laboratory system, center-of-mass system, direct and sequential processes, motion of the decaying compound nucleus.

\section{INTRODUCTION}

We shall study the interference phenomena when two particles are simultaneously detected (in a sense that will be specified below) in the nuclear reactions with three nuclei (particles) in the final channel. Earlier they have been considered by us for two-particle channels in the stationary theory (see, for instance, [1, 2]) and in the space-time description (see, for example, [3]).

\section{THE SPACE-TIME DESCRIPTION OF THE INTER- FERENCE BETWEEN DIFFERENT MECHANISMS WITH THREE NUCLEI IN THE FINAL CHANNEL}

The original idea was presented by Podgoretskij and Kopylov [4] for the two-particle emission (evaporation) from heavy nuclei. Here we consider the interference between prompt direct and delayed resonance processes in reaction of the type

$\mathrm{x}+\mathrm{X} \rightarrow \mathrm{y}+\mathrm{z}+\mathrm{U}$.

In Fig. (1a, b) two possible mechanisms for reaction (1) are pictorially represented.

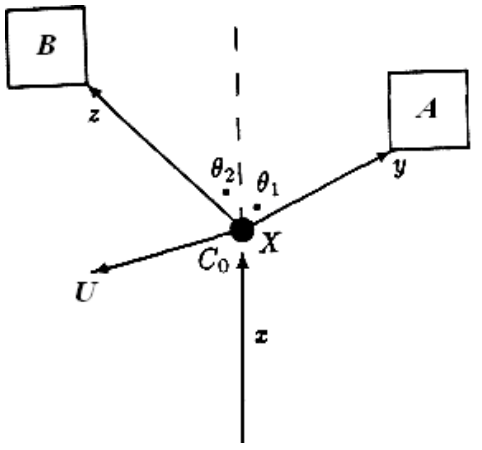

Fig. (1a). Direct process reaction channel.

*Address correspondence to this author at the Institute for Nuclear Research of NASU, Kiev-0650, Ukraine; Tel: 0038044 5255101; Fax: 0038044 5254463; E-mail: olkhovsky@mail.ru

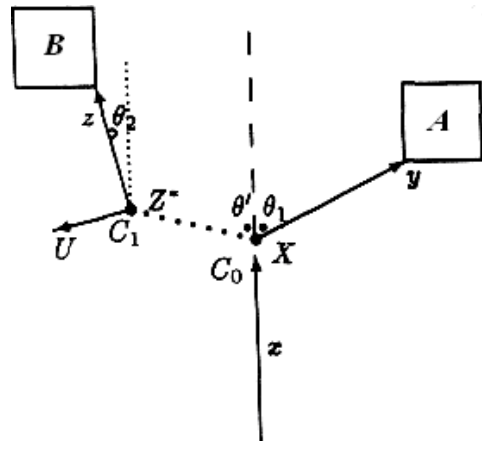

Fig. (1b). Sequential process reaction channel.

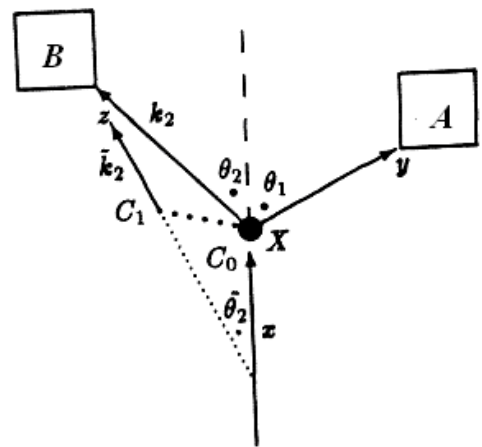

Fig. (1c). Simultaneous representation of direct and sequential processes.

The symbols $\boldsymbol{A}$ and $\boldsymbol{B}$ enclosed in boxes stand for detectors located at macroscopic distances $r_{1}$ and $r_{2}$ from the scattering point $C_{0}$. In Fig. (1a) the direct (like quasi-free or so called one and two step direct) process of simultaneous prompt emission at point $C_{0}$ of all the three final particles is described. Fig. (1b) presents delayed successive decay process with emission of particle $y$ and formation of an intermediate excited nucleus $Z^{*}$ which subsequently decays into $z$ and $U$ at point $C_{1}$, according the reaction

$\mathrm{x}+\mathrm{X} \rightarrow \mathrm{y}+\mathrm{Z}^{*}, \mathrm{Z}^{*} \rightarrow \mathrm{z}+\mathrm{U}$ 
In Fig. (1c) the superposition of the direct and the sequential emission of one from the final particle is displayed in the same picture. For macroscopic distances and under the condition specified below angles. $\theta_{2}$. and $\tilde{\theta}_{2}$ as well as impulses $\boldsymbol{k}$ and $\boldsymbol{k}_{2}$ can be considered practically coincident.

The asymptotic wave packet, near detectors $\boldsymbol{A}$ and $\boldsymbol{B}$ can be described by the following expression:

$$
\begin{aligned}
& \Psi_{a b}\left(r_{1}, r_{2} \rightarrow \infty\right) \rightarrow C \times \int d \mathrm{k}_{x} g_{i}\left(\mathrm{k}_{x}\right) \int d \mathrm{k}_{2} g_{f, 2}\left(\mathrm{k}_{2}\right) \int d \mathrm{k}_{1} f_{f, 1}\left(\mathrm{k}_{1}\right) \times \\
& \times \int d \mathrm{k}_{3} \delta\left(E_{i}-E_{f}\right) \delta\left(\mathrm{K}_{i}-\mathrm{K}_{f}\right)\left[f_{d i r}^{(L)}\left(E_{1}, E_{2}, E_{3}, \theta_{1}, \theta_{2}, \theta_{3}\right) e^{\left(\sum_{j=1}^{j i k_{j} r_{j} c_{0}}\right.}+\right. \\
& \left.\frac{f_{Z^{*}}^{(L)}\left(E_{1}, E_{2}, E_{3}, \theta_{1}, \theta_{2}, \theta_{3}\right)}{\varepsilon_{Z}^{*}-\varepsilon_{r e s, Z}+i \Gamma_{Z} / 2} e^{\left(i k_{1} r_{c_{0}}+\sum_{j=2}^{j=3} i r_{j c_{1}}\right)}\right] e^{-i E_{j} i / \hbar} .
\end{aligned}
$$

In this equation $C$ is a normalization constant, $g_{i}, g_{f, 1}, g_{f, 2}$ are amplitude weight factors describing the impulse spread of the incident particle $\mathrm{x}$ and that of the final particles $\mathrm{y}$ and $\mathrm{z}$ due to detectors resolution,

$f_{d i r}^{(L)}=\sqrt{J_{C \rightarrow L}} f_{d i r}^{(C)}$

and

$f_{Z^{*}}^{(C)}=\sqrt{J_{R \rightarrow C} J_{C \rightarrow L}} f_{x y}^{(C)} \Gamma_{Z}^{(C)}$

are the amplitudes for direct and sequential processes (the subscriptions $L$ and $C$ refer to laboratory and center of mass system, respectively), $f_{x y}^{(C)}$ and $\Gamma_{Z}^{(C)}$ being the amplitude of the first step direct process $x+X \rightarrow y+Z^{*}$ and the reducedwidth amplitude of the decay process $Z^{*} \rightarrow z+U$ respectively; $\varepsilon_{Z}^{*}, \varepsilon_{r e s, Z}$ and $\Gamma_{Z}$ are the excitation energy, the energy and total width of the resonant state of the nucleus $Z^{*}$; $J_{R \rightarrow C}$ and $J_{C \rightarrow L}$ are the Jacobians of the coordinate transformations from the Recoil system to the Center-ofMass (C.M.) one and from the C.M. system to the Laboratory one, respectively; $r \mathrm{~km}$ are the distances from points $m\left(m=C_{0}, C_{\mathrm{i}}\right)$ to particles $k$ (with $k=1,2,3$ corresponding to $y, z, U) ; E_{i}, \boldsymbol{k}_{i}$ and $E_{f}, \boldsymbol{k}_{f}$ the total energies and impulses in the initial and final channels respectively; $E_{j}$ $=\hbar^{2} k_{j}^{2} / 2 m_{j}$ is the kinetic energy of $j$-th particle, $\theta_{j}$ and $\boldsymbol{k}_{j}$ being the angle of motion (relative to beam, i.e. incident particle $x$, direction) and the wave vector of particle $j$, respectively. In expression (3) $\delta\left(E_{i}-E_{f}\right)$ and

$\delta\left(\mathrm{K}_{i}-\mathrm{K}_{f}\right)$ take care of energy and impulse conservation. Expression (3) is written on the bases of the general formalism described in [5] with application of the asymptotic stationary functions introduced in $[1,2]$ and taking into account particle $U$ explicitly. For the sake of simplicity the factor $r_{1 C_{0}}^{-1} r_{2 C_{0}}^{-1} r_{3 C_{0}}^{-1}$ has been omitted as well as spin and internal coordinates.

The factor $e-i E_{f} t / \hbar$ can be rewritten as
$e^{-i\left(E_{1}+E_{2}+E_{3}\right)} \frac{t}{\hbar} e^{-i E_{f}^{\prime} \frac{t}{\hbar}}$

and the first three factors of the expression (3), combined with the factor (6), can be formally put in the integrals of eq. (3) as follows:

$\int d \mathrm{k}_{1} g_{f, 1} e^{i k_{1} r_{1 m}-i E_{1} \frac{i}{k}} \ldots$

$\int d \mathrm{k}_{2} g_{f, 2 i} e^{i k_{1} r_{2 m}-i E_{2} \frac{i}{k}} \ldots$

$\int d \mathrm{k}_{3} g_{f, 3 i} e^{i k_{i} r_{3 m}-i E_{3} \frac{i}{k}} \ldots$

In order to perform the previous integrals a transformation from variables $k_{1,2,3}$ to variables

$\boldsymbol{y}_{1,2,3}=\left(\frac{i \hbar t}{m_{1,2,3}}\right)^{1 / 2}\left(\mathrm{k}_{1,2,3}^{0}-\frac{m_{1,2,3} \mathrm{r}_{1,2,3}}{\hbar t}\right)$

is useful. Here only projections of $\boldsymbol{k}_{1,2,3}$ over the mean vectors $\mathbf{k}_{1,2,3}^{0} \equiv<\boldsymbol{k}_{1,2,3}>$ are taken, the other components of $\boldsymbol{k}_{1,2,3}$ remaining in other parts of (3). The factor $g_{f 1,2}$ can be assumed to have the form

$g_{f 1,2} \approx \frac{c_{1,2}}{E_{1}-E_{1,2}^{0}-i \Delta E}$

and $\Delta E$ to be very small $\left(\Delta E<<\Gamma_{Z}\right)$, as well as the energy spread of the incident particle $x$. Using a known result for a similar calculation (see, for instance, $[6,7]$ ), the wave function becomes

$\Psi_{a b} \approx 0$

for

$$
\begin{aligned}
& \mathrm{t}<\mathrm{t}_{\mathrm{i}}+\frac{r_{1 C_{0}}}{v_{1}^{0}}, \mathrm{t}<\mathrm{t}_{\mathrm{i}}+\frac{r_{2 C_{0}}}{v_{2}^{0}}, \mathrm{t}<\mathrm{t}_{\mathrm{i}}+\frac{r_{3 C_{0}}}{v_{3}^{0}}, \\
& \mathrm{t}<\mathrm{t}_{\mathrm{i}}+\tau+\frac{r_{2 C_{1}}}{v_{2}^{0}}, \mathrm{t}<\mathrm{t}_{\mathrm{i}}+\tau+\frac{r_{3 C_{1}}}{v_{3}^{0}}
\end{aligned}
$$

and

$$
\begin{aligned}
& \Psi \propto C \times e^{-i E_{t}^{0} t \hbar} \times
\end{aligned}
$$

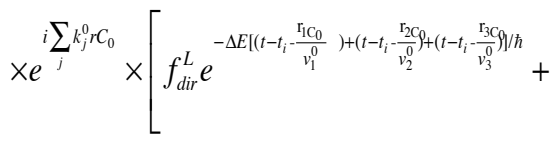

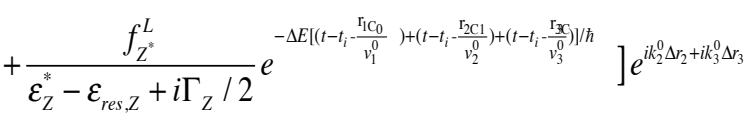

for

$\mathrm{t}>\mathrm{t}_{\mathrm{i}}+\frac{r_{1 C_{0}}}{v_{1}^{0}}, \mathrm{t}>\mathrm{t}_{\mathrm{i}}+\frac{r_{2 C_{0}}}{v_{2}^{0}}, \mathrm{t}>\mathrm{t}_{\mathrm{i}}+\frac{r_{3 C_{0}}}{v_{3}^{0}}$, 
$\mathrm{t}>\mathrm{t}_{\mathrm{i}}+\tau+\frac{r_{2 C_{1}}}{v_{2}^{0}}, \mathrm{t}>\mathrm{t}_{\mathrm{i}}+\tau+\frac{r_{3 C_{1}}}{v_{3}^{0}}$

Here $\mathbf{v}_{1,2,3}^{0}=\hbar \mathrm{k}_{1,2,3}^{0} / m_{1,2,3}$, the initial time $t_{i}$ is defined by the phase of the amplitude weight factor $g_{i}$; and the mean time $\tau$ of the nucleus $Z^{*}$ motion before its decay is given by the well known expression:

$$
\tau=\frac{\hbar \Gamma_{Z} / 2}{\left(\varepsilon_{Z}^{*}-\varepsilon_{r e s, Z}\right)^{2}+\Gamma_{Z}^{2} / 4}
$$

and

$\Delta r_{2,3}=V_{\perp(2,3)} \tau$,

$V_{\perp(2,3)}$ being the projection of the velocity of the nucleus $Z^{*}$ onto the direction of $\boldsymbol{k}_{2,3}$. The energy spread for particle $U$ is of the order $\Delta E$, according to energy-impulse conservation.

Interference phenomena can occur only in case of simultaneous arrival (within the time resolution of the detectors) of particles $y$ and $z$ on $A$ and $B$. The coincidencerate intensity is described by a time integration of

$\Psi_{a b}^{*} \hat{j}_{1} \hat{j}_{2} \Psi_{a b}$

$\left(\hat{j}_{1,2}\right.$ being the flux probability density operator for particles $y$ and $z$ ) over a time interval $\Delta T$, which is great with respect to the time extension of the wave packets, and a spatial integration over particle $U$ coordinates,

i.e.:

$P \approx \int_{t_{\min }}^{\infty} d t \int_{r_{3 \min }}^{r_{3 \max }} d r_{3} \Psi_{a b}^{*} \hat{j}_{1} \hat{j}_{2} \Psi_{a b} \propto \int_{t_{\min }}^{\infty} d t \int_{0}^{v_{3}^{0}\left(t-t_{i}-\frac{r_{3 c_{0}}}{v_{3}^{0}}\right)} d r_{3}\left|\Psi_{a b}\right|^{2}$,

where $t_{\min }$ is the smallest value among

$\mathrm{t}_{\mathrm{i}}+\frac{r_{1 C_{0}}}{v_{1}^{0}}, \mathrm{t}_{\mathrm{i}}+\frac{r_{2 C_{0}}}{v_{2}^{0}}, \mathrm{t}_{\mathrm{i}}+\frac{r_{3 C_{0}}}{v_{3}^{0}}$

$\mathrm{t}_{\mathrm{i}}+\tau+\frac{r_{2 C_{1}}}{v_{2}^{0}}, \mathrm{t}_{\mathrm{i}}+\tau+\frac{r_{3 C_{1}}}{v_{3}^{0}}$

$r_{3 \max }$ is the maximum between $v_{3}^{0}\left(t-t_{i}-\left(\frac{r_{3 C_{1}}}{v_{3}^{0}}\right)\right)$ and $v_{3}^{0}\left(t-t_{i}-\tau-\frac{r_{3 C_{1}}}{v_{3}^{0}}\right), \quad r_{3 \min } \rightarrow 0$ for ordinary small wave packets.

Under standard experimental conditions, i.e. when

$\Delta E \tau / \hbar<<1$

and

$\delta t=\frac{r_{l}}{v_{l}^{0}}-\frac{r_{m}}{v_{m}^{0}}<\Delta T,(l, m=1,2,3, l \neq m)$

( $\Delta T$ is the time resolution of the coincidence scheme), it is possible to write
$\mathrm{P}=\mathrm{P}_{0}+\mathrm{P}_{1},(18)$

$P_{0}=\left|f_{\text {dir }}^{L}\right|^{2}+\frac{\left|f_{Z^{*}}^{L}\right|^{2}}{\left(\varepsilon_{Z}^{*}-\varepsilon_{r e s, Z}\right)^{2}+\Gamma_{Z}^{2} / 4}$

and

$P_{1}=2\left|f_{\text {dir }}^{L} \frac{f_{Z^{*}}^{L}}{\varepsilon_{Z}^{*}-\varepsilon_{r e s, Z}+i \Gamma_{Z} / 2}\right| \cos \Phi$

(in arbitrary units), where

$\Phi=\delta+\beta+\phi$

$\delta=\arg \left(f_{Z^{*}}^{L}\right)-\arg \left(f_{d i r}^{L}\right)$,

$\beta=\arg \left(\varepsilon_{Z}^{*}-\varepsilon_{r e s, Z}+i \Gamma / 2\right)^{-1}$,

$\phi=k_{2}^{0} \Delta r_{2}+k_{3}^{0} \Delta r_{3}$,

$\Delta r_{2,3}$ being defined by (14)

The obtained results (18)-(20), with the incoherent sum $P_{0}$, the interference term $P_{1}$ and the phase $\Phi$, do evidently generalize the results, obtained somewhat earlier by us in $[3$, 8 ] for collisions with two-particle channels. Comparing these results with that obtained in a stationary model $[1,2]$, the latter ones are confirmed by the present self-consistent space-time approach in the limit $\Delta E<<\Gamma_{\mathrm{Z}}$. The same conclusion is valid for the cases in which two intermediate excited nuclei are formed, i.e.

$x+X \rightarrow\left\{\begin{array}{l}y+Z^{*} \rightarrow y+z+U \\ z+Y^{*} \rightarrow z+y+U\end{array}\right.$,

under the conditions $\Delta E<<\Gamma_{Y}$, and $\Delta E<<\Gamma_{Z}$.

\section{CONCLUSIONS}

The results (18)-(20) are firstly obtained in the spacetime description of the interference between different (direct and sequential, containing the decaying compound-nucleus) mechanisms with three nuclei in the final channel. They are the clear generalization of the results for nucleon-nucleus and nucleus-nucleus collisions with two-particle channels, presented in [7, 8], and can be easily generalized for the cases in which two intermediate excited (compound) nuclei are formed. Moreover in the limit $\Delta E / \Gamma_{Z} \rightarrow 0$ they factually pass to the correspondent stationary-model results as presented in $[1,2]$.

Finally, it is rather perspective and really topical to develop the much more complete approach to interference phenomena between the direct and various sequential processes in complex nuclear reactions (in particular, for application to a large amount of experimental data, reviewed in [9]).

\section{ACKNOWLEDGEMENTS}

The authors are thankful to Prof. G. Giardina and Dr. N.V. Eremin for the useful discussions.

\section{REFERENCES}

[1] Olkhovsky VS, Zaichenko AK. About the influence of space-time separations between sources of $\alpha$-particle emission in the reaction 
$\mathrm{p}+{ }^{11} \mathrm{~B} \rightarrow 3 \alpha$ on interference phenomena in their spectra. Phys Lett B 1991; 272: 183-5.

[2] D'Arrigo A, Fazio G, Giardina G, et al. On interference effects in light-ion nuclear reactions with three particles in the final state. Progress Theor Phys 1992; 87: 1359-65.

[3] Eremin NV, Giardina G, Olkhovsky VS, Omelchenko SA. Temporal description of interference phenomena in nuclear reactions with two-particle channels. Mod Phys Lett 1994; 9: 284956.

[4] Kopylov GI, Podgoretskij MI. Interference effects in tworesonance system. Sov J Nucl Phys 1972; 15: 219-23.

[5] Goldberger ML, Watson KM. Collision Theory Wiley J \& Son Inc.,1964.
[6] Baz AI, Zel'dovich Ya B, Perelomov AM. Scattering, reaction in nonrelatinatic qvantwn mechanic. Jerusalem, 1969.

[7] Rosenfeld L. Time evolution of the scattering process. Nucl Phys 1965; 70: 1-27.

[8] Olkhovsky VS, Dolinska ME, Omelchenko SA, Romanyuk MV. New developments in the tunneling and time analysis of lowenergy nuclear processes. Internat J Mod Phys E 2010; 19: N5-6: 1212-9.

[9] Keeley N,Alamanos N, Kemper KW, Rusek K. Elastic scattering and reactions of light exotic beams. Prog Part Nucl Phys 2009; 63: 396-447.

(c) Olkhovsky and Omelchenko; Licensee Bentham Open.

This is an open access article licensed under the terms of the Creative Commons Attribution Non-Commercial License (http://creativecommons.org/licenses/bync/3.0/) which permits unrestricted, non-commercial use, distribution and reproduction in any medium, provided the work is properly cited. 